where $K_{\sigma}$ is the Minkowski force on the particle

$$
K_{\sigma}=\int\left(k_{\sigma} u_{4} / i c\right) d V
$$

The mass $m$ though invariant in a Lorentz transformation is not necessarily a constant of the motion, even if the density $\rho$ of the particle is constant, since $m$ includes the thermodynamic internal energy [see Eq. (34)]. Included in the Minkowski force is a term which predicts a force on a particle when it exists in a temperature gradient, a force associated with the entropy the particle possesses. If $\bar{\delta}_{\sigma \tau} \partial_{\tau} T$ is constant throughout the volume of the particle, then this force is $-\delta \bar{\delta}_{\sigma \tau} \partial_{\tau} T$, where $S$ is the total entropy of the particle.

Equation (25) may be written in a form which facilitates comparison with the methods of field theory, namely,

$$
\partial_{\sigma} \rho u_{\sigma} u_{\tau}=\partial_{\sigma} T_{\sigma \tau}
$$

where

$$
T_{\sigma \tau}=-\left(1 / c^{2}\right)\left[\rho(A+T S) u_{\sigma} u_{\tau}+u_{\sigma} Q_{\sigma}+u_{\tau} Q_{\sigma}\right]+\phi_{\sigma \tau} .
$$

Equation (84) describes motion of a "naked" element, i.e., an element devoid of thermodynamic structure, through a field described by the energy-momentum tensor $T_{\sigma \tau}$. If we take $T_{\sigma \tau}$ to be the electromagnetic tensor,

$$
T_{\sigma \tau}=(1 / 4 \pi)\left(F_{\sigma \rho} F_{\rho \tau}+\delta_{\sigma \tau} F_{\rho \nu}{ }^{2} / 4\right),
$$

where $F_{\sigma \tau}=\partial_{\sigma} A_{\tau}-\partial_{\tau} A_{\sigma}$ and $A_{\sigma}$ is the four-vector potential, then $Q_{\sigma}{ }^{0}$ coincides with the Poynting flux, the energy density $\rho U=\left(E^{02}+H^{02}\right) / 8 \pi$, where $E^{0}$ and $H^{0}$ are the electric and magnetic intensities in the rest frame, and the stress tensor

$$
\begin{aligned}
4 \pi \phi_{\sigma \tau}=-\left(F_{\sigma \rho}+u_{\sigma} u_{\mu} F_{\mu \rho} / c^{2}\right)\left(F_{\tau \rho}+u_{\tau} u_{\nu} F_{\nu \rho} / c^{2}\right) \\
+\bar{\delta}_{\sigma \tau} F_{\rho \nu}{ }^{2} / 4,
\end{aligned}
$$

which reduces in the rest frame to the maxwell stress tensor in its nine space components and to zero in its fourth row and column.

\title{
Bound States in Quantum Field Theory
}

\author{
Murray Gell-Mann and Francis Low \\ Institute for Advanced Study, Princeton, New Jersey
}

(Received June 13, 1951)

\begin{abstract}
The relativistic two-body equation of Bethe and Salpeter is derived from field theory. It is shown that the Feynman two-body kernel may be written as a sum of wave functions over the states of the system. These wave functions depend exponentially on the energies of the states to which they correspond and therefore provide a means of calculating energy levels of bound states.
\end{abstract}

\section{INTRODUCTION}

SEVERAL attempts have been made to calculate the energy levels of bound systems of two particles that interact through a quantized field. The standard $\operatorname{method}^{1}$ has been to calculate an effective potential energy function and to insert that function into some two-particle Schroedinger or Dirac equation. In a case where the major effects of the interaction are obviously in the nonrelativistic region (e.g., the hydrogen atom), such a procedure seems reasonable (although even here higher order effects may not be describable by a potential). ${ }^{2}$ However, in the treatment of nuclear problems, one may have to deal with specifically relativistic interactions and singular forces for which methods successful in the atomic domain may fail entirely.

Recently, Dancoff ${ }^{3}$ has used an approximate method based directly on field theory. He has solved the

\footnotetext{
${ }^{1}$ G. Breit, Phys. Rev. 34, 553 (1929); Yukawa, Sakata, Kobayashi, and Taketani, Proc. Phys.-Math. Soc. Japan 20, 720 (1938)

2 Y. Nambu, Prog. Theor. Phys. 5, 614 (1950).

${ }^{3}$ S. M. Dancoff, Phys. Rev. 78, 382 (1950).
}

Schroedinger equation for the state vector with the requirement that it contain no particle-pairs and only one field quantum. The formal extension of his method to include higher approximations is difficult on account of the necessity of separating divergences in a noncovariant way. Furthermore, it appears impossible in his framework to make use of the elegant techniques developed by Feynman ${ }^{4}$ and Dyson. ${ }^{5}$

Bethe and Salpeter have proposed an equation ${ }^{6}$ for a two-body "wave function"; their equation is covariant in form and permits the separation of divergences as in the $S$-matrix theory. Their reasoning, however, is based on an analogy to that in Feynman's "Theory of positrons"4 and the demonstration of equivalence to

${ }^{4}$ R. P. Feynman, Phys. Rev. 76, 749 (1949); Phys. Rev. 76, 769 (1949).

' F. J. Dyson, Phys. Rev. 75, 486 (1949); Phys. Rev. 75, 1736 (1949).

${ }^{6} \mathrm{H}$. A. Bethe and E. E. Salpeter, Phys. Rev. 82, 309 (1951). We are indebted to Drs. Bethe and Salpeter for communicating their results to us prior to publication. We understand that this equation has been treated by Schwinger in his lectures at Harvard. 
conventional field theory is incomplete. Our purpose is to provide such a demonstration.

The starting point of the argument of Bethe and Salpeter is the consideration of the so-called Feynman two-body kernel, and the proof that its usual power series expansion can be re-expressed as an integral equation. We will begin in a similar fashion; however, we will exhibit all quantities of which we make use (in particular, the wave function) as matrix elements of field operators, and derive their properties in the conventional way.

\section{GENERAL THEORY}

For simplicity we will consider a proton field $\psi$ and a neutron field $\phi$ coupled by a neutral scalar meson field, $A$. (The generalization to other cases is direct.) All symbols unless otherwise labeled refer to the Heisenberg representation. Let us denote by $P_{\mu}$ the total energymomentum four vector of the interacting fields; $P_{\mu}$ acts as a displacement operator in the sense that for any function $F$ of the field-variables at the space-time point $x$ the equation

$$
\partial F / \partial x_{\mu}=i\left[F, P_{\mu}\right]
$$

holds. ${ }^{7}$ We may choose a complete set $\Psi_{n}$ of statevectors such that

$$
P_{\mu} \Psi_{n}=p_{\mu}{ }^{n} \Psi_{n} .
$$

Each $\Psi_{n}$ will then describe a stationary state.

The component $P_{0}$ can be written as the hamiltonian function $H$ of the field variables and their conjugate momenta. Although $H$ is independent of time, it is convenient to separate it into two time-dependent parts, $H_{0}(t)$ and $H_{I}(t)$, where $H_{0}(t)$ is the sum of the free-field hamiltonians. We shall use $H_{0}$ to define an interaction representation that reduces to the Heisenberg representation at a finite time, which we shall take to be $t=0$. For any time dependent operator $O(t)$ the corresponding operator $\mathbf{O}(t)$ in the interaction representation is given by

$$
\mathbf{0}(t)=\exp \left(i H_{0}(0) t\right) O(0) \exp \left(-i H_{0}(0) t\right) .
$$

According to Eq. (1), the time-dependence of the Heisenberg operators is of the form,

$$
O(t)=\exp (i H t) O(0) \exp (-i H t)
$$

so that Eq. (3) can be written

where

$$
\mathbf{0}(t)=U(t, 0) O(t) U^{-1}(t, 0),
$$

$$
U(t, 0)=\exp \left(i H_{0}(0) t\right) \exp (-i H t)
$$

and thus satisfies the familiar differential equation,

$$
i d U(t, 0) / d t=\mathbf{H}_{I}(t) U(t, 0),
$$

with the boundary condition

$$
U(0,0)=1
$$

\footnotetext{
${ }^{7}$ We take $\hbar=c=1$.
}

We shall make extensive use of the true vacuum state $\Psi_{0}$ and the vacuum of free particles, $\Phi_{0}$. The former is the eigenstate of $H$ with the lowest energy $p_{0}{ }^{0}=E_{0}$. The latter is the eigenstate of $\mathbf{H}_{0}=H_{0}(0)$ with the lowest eigenvalue $\epsilon_{0}$.

It will be necessary to express $\Psi_{0}$ in terms of $\Phi_{0}$ and operators of the interaction representation. There are two standard methods of handling that problem. The first is to introduce the interaction representation at a time $t=-\infty$ rather than at $t=0$ as we have done; it is then assumed that at $-\infty$ the coupling constant vanishes, $H$ and $\mathbf{H}_{0}$ are identical, and thus $\Psi_{0}=\Phi_{0}$. It will become clear that such a procedure does indeed yield correct results, but it would be inconsistent to base a discussion of stationary states on a physical assumption of the variability of charge. The second method is that of stationary-state perturbation theory; the interaction representation can be defined as above at $t=0$, and $\Psi_{0}$ expressed in the form,

$c \Psi_{0}=\left[1+\left(H_{0}-\epsilon_{0}\right)^{-1}(1-V)\left(H_{I}(0)-E_{0}+\epsilon_{0}\right)\right]^{-1} \Phi_{0}$,

where $V$ is the projection operator on the state $\Phi_{0}$ and $c$ is a normalization constant. We are then free to write Eq. (9) in terms of integrals over time (as a parameter), for example in terms of the $U$ matrix considered as a solution of Eq. (7). We shall show in the Appendix that Eq. (9) may be replaced by the formal equation,

$$
c \Psi_{0}=U^{-1}( \pm \infty, 0) \Phi_{0} /\left(\Phi_{0}, U^{-1}( \pm \infty, 0) \Phi_{0}\right) .
$$

In fact, both the numerator and denominator of Eq. (10) are indeterminate on account of the presence of a phase factor $\exp (i \infty)$; however, the quotient is well defined in terms of a suitable limiting process, such as we shall exhibit in the Appendix, which does indeed involve turning the charge on and off infinitely slowly, but only as part of a mathematical prescription for solving the stationary state problem.

\section{THE INTEGRAL EQUATION FOR THE KERNEL}

We define the Feynman two-body kernel as follows:

$K\left(x_{1} x_{2} ; x_{3} x_{4}\right)=\epsilon\left(\Psi_{0}, P\left[\psi\left(x_{1}\right) \phi\left(x_{2}\right) \bar{\psi}\left(x_{3}\right) \bar{\phi}\left(x_{4}\right)\right] \Psi_{0}\right)$,

where $P$ is Dyson's time-ordering operator and $\epsilon$ is -1 if the permutation of the times (1234) induced by $P$ is even, +1 if it is odd. Introducing the interaction representation at time $t=0$, and making use of Eqs. (5) and (10), we have, for a typical order of the times $\left(t_{1} \geq t_{2} \geq t_{3} \geq t_{4}\right)$,

$$
\begin{aligned}
K\left(x_{1} x_{2} ; x_{3} x_{4}\right)= & -\left(\Phi_{0}, U(+\infty, 0) U^{-1}\left(t_{1}, 0\right) \psi\left(x_{1}\right)\right. \\
& \times U\left(t_{1}, 0\right) U^{-1}\left(t_{2}, 0\right) \phi\left(x_{2}\right) \cdots \\
& \left.\times \bar{\phi}\left(x_{4}\right) U\left(t_{4}, 0\right) U^{-1}(-\infty, 0) \Phi_{0}\right) / \\
& \left(\Phi_{0}, U(\infty, 0) U^{-1}(-\infty, 0) \Phi_{0}\right),
\end{aligned}
$$

where the ratio is necessary to eliminate the constant $c$, and terms of the form $\left(\Phi_{0}, U^{-1}( \pm \infty, 0) \Phi_{0}\right)$ have canceled. It is clear that

$$
U(t, 0) U^{-1}\left(t^{\prime}, 0\right)=U\left(t, t^{\prime}\right)
$$




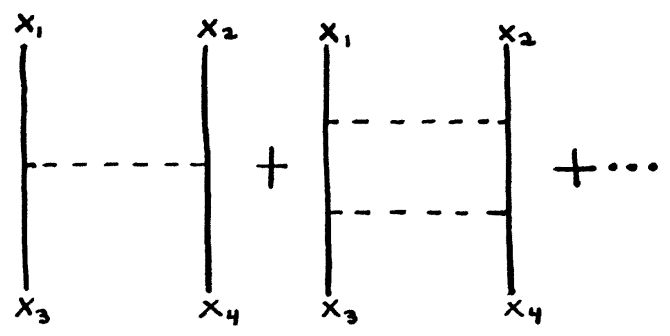

FIG. 1. Typical Feynman diagrams.

where

$$
i \partial U\left(t, t^{\prime}\right) / \partial t=\mathbf{H}_{I}(t) U\left(t, t^{\prime}\right)
$$

and

$$
U\left(t^{\prime}, t^{\prime}\right)=1 .
$$

$U\left(t, t^{\prime}\right)$ may be expressed as a power series:

$U\left(t, t^{\prime}\right)=\sum_{n=0}^{\infty} \frac{(-i)^{n}}{n !} \int_{t^{\prime}}^{t} d \tau_{1} \cdots d \tau_{n} P\left[\mathbf{H}_{I}\left(\tau_{1}\right) \cdots \mathbf{H}_{I}\left(\tau_{n}\right)\right]$

Using Eqs. (12), (13), and (15), we find $K=\boldsymbol{\epsilon}\left(\Phi_{0}, P\left[U(\infty,-\infty) \boldsymbol{\psi}\left(x_{1}\right) \boldsymbol{\phi}\left(x_{2}\right) \bar{\Psi}\left(x_{3}\right) \overline{\boldsymbol{\phi}}\left(x_{4}\right)\right] \Phi_{0}\right) /$

$$
\left(\Phi_{0}, U(\infty,-\infty) \Phi_{0}\right) \text {, }
$$

where

$$
\begin{aligned}
& P[U(\infty,-\infty) \psi \phi \overline{\boldsymbol{\Psi}} \overline{\boldsymbol{\phi}}] \\
& =\sum_{n=0}^{\infty} \frac{(-i)^{n}}{n !} \int_{-\infty}^{\infty} d \tau_{1} \cdots d \tau_{n} \\
& \quad \times P\left[\mathbf{H}_{I}\left(\tau_{1}\right) \cdots \mathbf{H}_{I}\left(\tau_{n}\right) \psi \boldsymbol{\phi} \overline{\mathbf{\Psi}} \overline{\boldsymbol{\phi}}\right] .
\end{aligned}
$$

From an expansion of Eq. (16) in powers of the coupling constant, it is clear that $K$ is equal to the twobody kernel as given by Feynman in terms of Feynman diagrams :

$$
\begin{aligned}
K(1,2 ; 3,4)= & S_{F}^{P}(1,3) S_{F}^{N}(2,4)-g^{2} \int_{-\infty}^{\infty} \int_{-\infty}^{\infty} d \omega_{5} d \omega_{6} \\
& \times S_{F}^{P}(1,5) S_{F}^{N}(2,6) \Delta_{F}(5,6) \\
& \times S_{F}^{P}(5,3) S_{F}^{N}(6,4)+\cdots,
\end{aligned}
$$

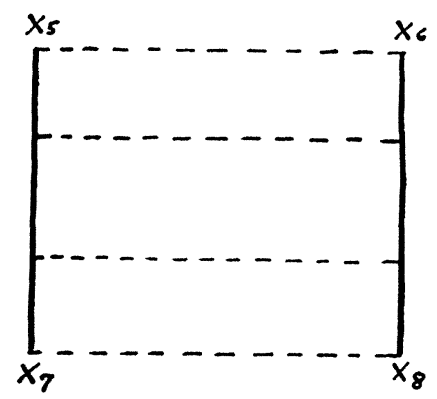

Fig. 2. An excluded Feynman diagram. (It can be decomposed into simpler diagrams.) where

$$
\begin{aligned}
S_{F}^{P}(x-y) & =\epsilon(x, y)\left(\Phi_{0}, P[\psi(x) \bar{\psi}(y)] \Phi_{0}\right), \\
S_{F}^{N}(x-y) & =\epsilon(x, y)\left(\Phi_{0}, P[\phi(x) \bar{\phi}(y)] \Phi_{0}\right), \\
\Delta_{F}(x-y) & =\left(\Phi_{0}, P[A(x) A(y)] \Phi_{0}\right) .
\end{aligned}
$$

Here, $\epsilon(x, y)$ is +1 if $t_{x}>t_{y}$ and -1 if $t_{x}<t_{y}$; and $d \omega$ is the four-dimensional volume element.

Bethe and Salpeter have shown, by summing the terms of Eq. (18) in a particular order, that the power series can be converted into an integral equation. All terms arising from diagrams of the type shown in Fig. 1 may be combined to give the integral equation

$$
\begin{array}{r}
K(1,2 ; 3,4) \cong S_{F}^{P}(1,3) S_{F}{ }^{N}(2,4)-g^{2} \int_{-\infty}^{\infty} d \omega_{5} d \omega_{6} \\
\times S_{F}^{P}(1,5) S_{F}^{N}(2,6) \Delta_{F}(5,6) K(5,6 ; 3,4) .
\end{array}
$$

The exact integral equation may be written

$K(1,2 ; 3,4)={S_{F}}^{P}(1,3) S_{F}{ }^{\prime N}(2,4)$

$$
\begin{array}{r}
-\int_{-\infty}^{\infty} d \omega_{5} d \omega_{6} d \omega_{7} d \omega_{8} S_{F}{ }^{P}(1,5) S_{F}{ }^{\prime N}(2,6) \\
\times G(5,6 ; 7,8) K(7,8 ; 3,4),
\end{array}
$$

where, in contrast with Eq. (19),

$$
S_{F}{ }^{P}(x-y)=\epsilon(x, y)\left(\Psi_{0}, P[\psi(x) \bar{\psi}(y)] \Psi_{0}\right),
$$

all symbols now referring to the Heisenberg representation. The interaction function $G$ is given in lowest order by

$$
G(5,6 ; 7,8)=g^{2} \Delta_{F}(5,6) \delta(5,7) \delta(6,8)
$$

and in general, in a given order, by the sum of all diagrams of that order that cannot be decomposed into simpler diagrams connected by one proton and one neutron line. For instance, the diagram in Fig. 2 is specifically excluded, its effect being already contained in Eq. (22). All nucleon self-energy parts may be omitted, since their effect is simply to substitute $S_{F}{ }^{\prime}$ for $S_{F}$ in every nucleon line occuring in $G$.

It is interesting to compare the power series (18) and the integral equation (23) for the kernel from the point of view of convergence. The power series is certainly badly divergent, in general. Looking at the integral equation, we see that there are three possible sources of such divergence of the series:

(a) Certain terms in the expansion of the interaction function $G$ and of $S_{F}{ }^{\prime}$ contain infinite integrals which are known to be canceled by renormalization procedures (see Dyson ${ }^{5}$ and Salam ${ }^{8}$ ).

(b) After renormalization, the expansion of $G$ may not converge. We are not prepared to discuss this matter, but will assume that $G$ is well defined.

(c) For sufficiently large $g$, the power series for $K$ will diverge merely on account of the failure of the Born approximation. ${ }^{9}$ In such a case, the integral equation will continue to have meaning; although the series has none.

${ }^{8}$ A. Salam, Phys. Rev. 82, 217 (1951).

${ }^{9}$ R. Jost and A. Pais, Phys. Rev. 82, 840 (1951). 


\section{THE INTEGRAL EQUATION FOR THE WAVE FUNCTION}

The connection of the kernel $K$ with the properties of the stationary states of the two-nucleon system may be exhibited in the following way: Let $t_{1}, t_{2}>t_{3}, t_{4}$. Then, using the completeness of the set of stationary states $\Psi_{n}$, we have from Eq. (11)

$$
\begin{aligned}
& \begin{aligned}
K(1,2 ; 3,4)=\sum_{n} & \left(\Psi_{0}, \epsilon(12) P\left[\psi\left(x_{1}\right) \phi\left(x_{2}\right)\right] \Psi_{n}\right) \\
& \times\left(\Psi_{n}, P\left[\bar{\psi}\left(x_{3}\right) \bar{\phi}\left(x_{4}\right)\right] \epsilon(43) \Psi_{0}\right),
\end{aligned} \\
& \text { or } \begin{aligned}
& K=\sum_{n} \chi_{n}(1,2) \bar{\chi}_{n}(3,4), \\
& \text { where } \quad \chi_{n}\left(x_{1}, x_{2}\right)=\left(\Psi_{0}, \epsilon(1,2) P\left[\psi\left(x_{1}\right) \phi\left(x_{2}\right)\right] \Psi_{n}\right)
\end{aligned} \\
& \text { and } \\
& \bar{\chi}_{n}\left(x_{3}, x_{4}\right)=\left(\Psi_{0}, \epsilon(4,3) \bar{P}\left[\psi\left(x_{3}\right) \phi\left(x_{4}\right)\right] \Psi_{n}\right)^{*} \beta_{N} \beta_{P} .
\end{aligned}
$$

Here $\bar{P}$ orders operators in the reverse sense to $P$ and $\beta_{N}$ and $\beta_{P}$ are the usual Dirac matrices.

Let us observe that $\chi_{n}$ possesses the symmetry properties of the state $\Psi_{n}$. For example, $\Psi_{n}$ is an eigenstate of the displacement operators $P_{\mu}$; and, therefore, a simultaneous displacement of the coordinates of both particles yields

$$
\begin{aligned}
\left(\frac{\partial}{\partial x_{1 \mu}}+\frac{\partial}{\partial x_{2 \mu}}\right) \chi_{n}=-i\left(\Psi_{0},\left[P_{\mu}, \epsilon P(\psi \phi)\right] \Psi_{n}\right) \\
=i\left(p_{\mu}{ }^{n}-p_{\mu}{ }^{0}\right) \chi_{n},
\end{aligned}
$$

where we have made use of Eq. (1) and of the fact that the differential operator commutes with $\epsilon$ and with $P$. Hence, we may write

$$
\chi_{n}=V^{-\frac{1}{2}} \exp \left[i\left(p_{\mu}{ }^{n}-p_{\mu}{ }^{0}\right) X_{\mu}\right] f_{n}(x),
$$

where $X$ is any center-of-gravity coordinate, $x=x_{1}-x_{2}$, and $V$ is the normalizing volume. Similarly, we have

$$
\bar{\chi}_{n}=V^{-\frac{1}{2}} \exp \left[-i\left(p_{\mu}{ }^{n}-p_{\mu}{ }^{0}\right) X_{\mu}\right] \bar{f}_{n}(x) .
$$

It can now be seen that a knowledge of the kernel is sufficient to determine the energy-levels of the system. However, it will obviously be advantageous to find an equation for the simpler quantity $\chi_{n}$; for that purpose we seek a method of isolating a single wave function from the sum that occurs in the kernel.

In the absence of interaction the wave functions $\chi_{n}$ possess properties of orthogonality and normalization which enable one to write, for $t<t_{1}, t_{2}$,

$$
\begin{aligned}
\int K\left(x_{1} x_{2} ; \mathbf{x}_{3}, t, \mathbf{x}_{4}, t\right) \beta_{N} \beta_{p} \chi_{n}\left(\mathbf{x}_{2}, t, \mathbf{x}_{4}, t\right) d \mathbf{x}_{3} d \mathbf{x}_{4} \\
=\chi_{n}\left(x_{1}, x_{2}\right), \quad(g=0)
\end{aligned}
$$

(where $\mathbf{x}$ stands for the spacelike part of $x$ and $d \mathbf{x}$ is the three-dimensional volume element); but, in general, such is not the case. However, we may always make use of the fact that wave functions of states of different energy are characterized by different exponential time dependences.
Consider a bound state, $\Psi_{\alpha}$. Its energy, barring accidental degeneracy, will differ from that of every other state possessing the same values of momentum, internal angular momentum, and other constants of the motion. If we set up the left-hand member of Eq. (32) for $n=\alpha$, general $g$, and $t<t_{1}, t_{2}$, we have, by Eqs. (26), (30), and (31),

$$
\begin{array}{r}
\int K\left(x_{1}, x_{2} ; \mathbf{x}_{3}, t, \mathbf{x}_{4}, t\right) \beta_{N} \beta_{P} \chi_{\alpha}\left(\mathbf{x}_{3}, t, \mathbf{x}_{4}, t\right) d \mathbf{x}_{3} d \mathbf{x}_{4} \\
=\sum_{n} \chi_{n}\left(x_{1}, x_{2}\right) V^{-1} \int d \mathbf{X} \exp \left[i\left(\mathbf{P}_{\alpha}-\mathbf{P}_{n}\right) \mathbf{X}\right. \\
\left.-i\left(E_{\alpha}-E_{n}\right) t\right] \int d \mathbf{x} f_{n}{ }^{*}(\mathbf{x}) f_{\alpha}(\mathbf{x}),
\end{array}
$$

where $f_{n}^{*}(\mathbf{x})$ is the hermitian conjugate of the column vector $f_{n}(\mathbf{x})$ (the times $t_{3}$ and $t_{4}$ both being equal to $t$ ). We may now eliminate all the $\chi_{n}$ but $\chi_{\alpha}$ by performing the following operation on (33): We take the limit $L$ of both members as $t \rightarrow-\infty$ in the sense that all oscillating terms are taken to approach zero. As an example of a linear operation of this kind, we might take

$$
\underset{t \rightarrow-\infty}{L} f(t)=\lim _{t \rightarrow-\infty} \frac{1}{|t|} \int_{2 t}^{t} f(\tau) d \tau .
$$

Then, we have

$$
\begin{array}{r}
\underset{t \rightarrow-\infty}{L} \int K\left(x_{1}, x_{2} ; \mathbf{x}_{3}, t, \mathbf{x}_{4}, t\right) \beta_{N} \beta_{P} \chi_{\alpha}\left(\mathbf{x}_{3}, t, \mathbf{x}_{4}, t\right) d \mathbf{x}_{3} d \mathbf{x}_{4} \\
=\chi_{\alpha}\left(x_{1}, x_{2}\right) P_{2}(\alpha),
\end{array}
$$

where

$$
P_{2}(\alpha)=\int d \mathbf{x} f_{\alpha}^{*}(\mathbf{x}) f_{\alpha}(\mathbf{x}) .
$$

The inner product has removed states degenerate with $\alpha$ from the sum by symmetry.

The relation (34) enables us to derive from Eq. (23) an integral equation for the bound state wave function $\chi_{\alpha}$. We have

$$
\begin{aligned}
& P_{2}(\alpha) \chi_{\alpha}\left(x_{1}, x_{2}\right)=\underset{t \rightarrow-\infty}{L} \int S_{F}{ }^{P}\left(x_{1} ; \mathbf{x}_{3}, t\right) \\
& \times S_{F}{ }^{\prime}\left(x_{2}, \mathbf{x}_{4}, t\right) d \mathbf{x}_{3} d \mathbf{x}_{4} \beta_{P} \beta_{N} \chi_{\alpha}\left(\mathbf{x}_{3}, t, \mathbf{x}_{4}, t\right) \\
& -\underset{t \rightarrow-\infty}{L} \int d \omega_{5} d \omega_{6} d \omega_{7} d \omega_{8} S_{F}{ }^{P}(1,5) \\
& \times S_{F}{ }^{\prime N}(2,6) G(5,6 ; 7,8) \int d \mathbf{x}_{3} d \mathbf{x}_{4} K\left(7,8 ; \mathbf{x}_{3}, t, \mathbf{x}_{4}, t\right) \\
& \quad \times \beta_{N} \beta_{P} \chi_{\alpha}\left(\mathbf{x}_{3}, t, \mathbf{x}_{4}, t\right) .
\end{aligned}
$$

Let us examine the second term on the right of Eq. (35). As $t \rightarrow-\infty$, the region of integration in which $t_{7}$ or $t_{8}$ is less than $t$ gives a vanishingly small contribution to 
the integral. We may therefore employ Eq. (34) and obtain for the second term the value

$$
\begin{aligned}
-P_{2}(\alpha) \int d \omega_{5} d \omega_{6} d \omega_{7} d \omega_{8} S_{F}{ }^{\prime} P(1,5) S_{F}{ }^{N}(2,6) & \\
& \times G(5,6 ; 7,8) \chi_{\alpha}(7,8) .
\end{aligned}
$$

To evaluate the first term we note that, analogously to Eqs. (25) and (26), we can write $S_{F}{ }^{P} \cdot S_{F}{ }^{N}$ as a sum of products of neutron and proton wave functions; for a given momentum, these will never contain frequencies as low as $E_{\alpha}$. Thus, $L$, applied to the first term, gives zero; and we find as our final result the integral equation,

$$
\begin{aligned}
\chi_{\alpha}(1,2)=-\int d \omega_{5} d \omega_{6} d \omega_{7} d \omega_{8} S_{F}{ }^{P}(1,5) \\
\quad \times S_{F}{ }^{\prime}(2,6) G(5,6 ; 7,8) \chi_{\alpha}(7,8) .
\end{aligned}
$$

The eigenvalue problem of the mass of the bound system appears when we substitute Eq. (30) into Eq. (37).

The simplest covariant approximation to Eq. (37) is the analog of the approximate equation (22) for the kernel,

$$
\begin{array}{r}
\chi_{\alpha}(1,2)=-g^{2} \int d \omega_{5} d \omega_{6} S_{F}^{P}(1,5) S_{F}^{N}(2,6) \\
\times \Delta_{F}(5,6) \chi_{\alpha}(5,6) .
\end{array}
$$

The differential equation of which Eq. (38) is a particular integral,

$$
\begin{aligned}
\left(\gamma P_{\mu} \frac{\partial}{\partial x_{1 \mu}}+m_{P}\right)\left(\gamma N_{\nu} \frac{\partial}{\partial x_{2 \nu}}+m_{N}\right) \chi_{\alpha}(1,2) \\
=g^{2} \Delta_{F}(1,2) \chi_{\alpha}(1,2),
\end{aligned}
$$

has also been proposed by Nambu. ${ }^{2}$

The authors wish to express their gratitude to Dr. J. R. Oppenheimer and to the Institute for Advanced Study for their kind hospitality. They would also like to thank the members of the Institute for many interesting discussions.

\section{APPENDIX. THE VACUUM STATE}

In order to derive Eq. (10), we must first attach a rigorous meaning to the right-hand member. For convenience we will take it to mean

$$
\lim _{\alpha \rightarrow 0} U_{\alpha}{ }^{-1}( \pm \infty, 0) \Phi_{0} /\left(\Phi_{0}, U_{\alpha}^{-1}( \pm \infty, 0) \Phi_{0}\right)=\lim _{\alpha \rightarrow 0} X_{\alpha},
$$

where $U_{\alpha}(t, 0)$ satisfies the differential equation

$$
i d U_{\alpha}(t, 0) / d t=\mathbf{H}_{I}(t) U_{\alpha}(t, 0) \exp (-\alpha|t|)
$$

instead of Eq. (7)

It is well known that an expression such as the quotient $X_{\alpha}$ is calculated by the same rules (in terms of Feynman diagrams) as the numerator, except that all disconnected closed loops are omitted. Moreover, the resulting sum is known to be free of factors of the form $\exp (i a / \alpha)$ that are introduced by disconnected closed loop diagrams; thus in a finite theory the limit in Eq. (A1) exists.

For any finite $\alpha$ we may write

$$
\begin{aligned}
\Psi_{0}(\alpha)=U_{\alpha}^{-1}(-\infty, 0) \Phi_{0}=U_{\alpha}(0,-\infty) \Phi_{0} \\
=\sum_{n=0}^{\infty} \frac{(-i)^{n}}{n !} \int_{-\infty}^{0} d t_{1} \cdots d t_{n} \exp \left[\alpha\left(t_{1}+\cdots+t_{n}\right)\right] \\
\times P\left[\mathbf{H}_{I}\left(t_{1}\right) \cdots \mathbf{H}_{I}\left(t_{n}\right)\right] \Phi_{0}
\end{aligned}
$$

and

$$
\begin{aligned}
& \begin{aligned}
&\left(\mathbf{H}_{0}-\epsilon_{0}\right) \Psi_{0}(\boldsymbol{\alpha})= {\left[\mathbf{H}_{0}, U_{\alpha}(0,-\infty)\right] \Phi_{0} } \\
&= \frac{1}{i} \sum_{(n=1)}^{\infty} \frac{(-i)^{n}}{n !} \int_{-\infty}^{0} d t_{1} \cdots d t_{n} \exp \left[\alpha\left(t_{1}+\cdots+t_{n}\right)\right] \\
& \quad \times \sum_{l=1}^{n} \frac{\partial}{\partial t_{l}} P\left[\mathbf{H}_{I}\left(t_{1}\right) \cdots \mathbf{H}_{I}\left(t_{n}\right)\right] \Phi_{0}
\end{aligned} \\
& =-\sum_{(n=1)}^{\infty} \frac{(-i)^{n-1}}{(n-1) !} \int_{-\infty}^{0} d t_{1} \cdots d t_{n} \exp \left[\alpha\left(t_{1}+\cdots+t_{n}\right)\right] \\
& \frac{\partial}{\partial t_{1}} P\left[\mathbf{H}_{I}\left(t_{1}\right) \cdots \mathbf{H}_{I}\left(t_{n}\right)\right] \Phi_{0}
\end{aligned}
$$

But from (A5) we see that

$$
\left(\Phi_{0},\left(H-\epsilon_{0}\right) X_{\alpha}\right)=i \alpha g(\partial / \partial g) \log \left(\Phi_{0}, \Psi_{0}(\alpha)\right) .
$$

Thus, we have

$$
\left(H-\epsilon_{0}\right) \lim X_{\alpha}=\left(\Phi_{0},\left(H-\epsilon_{0}\right) \lim X_{\alpha}\right) \lim X_{\alpha}
$$

Moreover, $\left(\Phi_{0}, X_{\alpha}\right)$ is clearly 1 . Hence, $\lim X_{\alpha}$ is indeed $c \Psi_{0}$.

It may be of interest to note that the expressions (9) and (A1) can be compared directly. Performing the integrations in Eq. (A3), we have

$$
\begin{aligned}
& \Psi_{0}(\alpha)=\left[1-\left(H_{0}-\epsilon_{0}-i \alpha\right)^{-1} H_{I}(0)+\left(H_{0}-\epsilon_{0}-2 i \alpha\right)^{-1}\right. \\
&\left.\times H_{I}(0)\left(H_{0}-\epsilon_{0}-i \alpha\right)^{-1} H_{I}(0)+\cdots\right] \Phi_{0} .
\end{aligned}
$$

Substituting Eq. (A9) into Eq. (A1) and taking the limit, one may check the agreement with Eq. (9) to any desired order.

The treatment of $U^{-1}(+\infty, 0)$ is exactly analogous to that of $U^{-1}(-\infty, 0)$. 